\title{
TRANSPLANTE DE FÍGADO NO PROGRAMA DE TRATAMENTO FORA DE DOMICÍLIO NO ESTADO DE SERGIPE EM 2008. ANÁLISE DE DADOS CLÍNICOS E CUSTO
}

\author{
Liver transplantation in the out of home treatment program in the state of Sergipe in 2008. Clinical \\ analysis and costs.
}

\author{
Bianca Souza Leal', Tereza Virgínia Silva Bezerra Nascimento², João Augusto Guimarães Figueiredo³, \\ Alex Vianey Callado França².
}

\begin{abstract}
RESUMO
No Brasil, a maioria dos transplantes de fígado é realizada na região Sudeste. Nos estados em que não há serviço de transplante de fígado, como Sergipe, os pacientes usuários do Sistema Único de Saúde são encaminhados para outros estados e as despesas com deslocamento e ajuda de custo são financiadas pelo Programa de Tratamento Fora de Domicílio (TFD). Objetivo: Avaliar os principais aspectos dos pacientes cadastrados para transplante de fígado (pré- e pós-) e custos dos serviços financiados pelo TFD em Sergipe no ano de 2008. Método: Análise dos prontuários de 55 pacientes cadastrados no TFD em Sergipe que viajaram em 2008. Foram avaliados: idade, gênero, procedência, naturalidade, presença de cirrose e evolução com óbito em 2008. Compararam-se as características e custos gerados pelos pacientes, acompanhantes e doadores e ainda entre os pacientes transplantados e não-transplantados. Resultados: Predominaram pacientes do gênero masculino (63,6\%), com idades entre 12 e 59 anos (61,8\%) procedentes da capital - Aracaju/SE - (50,9\%) e do interior de Sergipe (49,1\%). Apresentaram cirrose $80 \%$ dos pacientes e $12,7 \%$ evoluíram para óbito em 2008. As principais causas da cirrose foram Hepatite $\mathrm{C}$ e Alcoolismo, com 15,9\% dos casos cada uma. Foram transplantados 30,9\% dos pacientes, sendo 76,5\% desses transplantes realizados em São Paulo. Os acompanhantes formaram o grupo que gerou maior despesa para o TFD, com gasto de R\$217.575,72, em um total de $\mathrm{R} \$ 442.088,43$ incluindo pacientes e doadores. O custo médio de cada paciente financiado pelo TFD foi de R\$ 8.037,97 e quando incluídas despesas adicionais, a média foi de $\mathrm{R} \$ 10.590,49$ por paciente. Os pacientes viajaram em média 4,76 vezes em 2008 e os transplantados geraram despesa 1,8 vezes maior do que os não-transplantados. Conclusão: Cirrose hepática foi a principal causa de indicação de transplante de fígado. Em 2008, o custo do programa foi de cerca de R \$ 8.000,00 por paciente, valor que poderia ser investido, em parte, na estruturação de serviço especializado no acompanhamento tanto no pré- quanto no pós-transplante no estado de origem do paciente.
\end{abstract}

Descritores: Transplante de Fígado, Gastos em Saúde, Sistema Único de Saúde.

\footnotetext{
Instituição:

${ }^{1}$ Curso de Medicina da Universidade Federal de Sergipe, Aracaju/SE

${ }^{2}$ Departamento de Medicina da Universidade Federal de Sergipe, Aracaju/SE

${ }^{3}$ Programa de Tratamento Fora de Domicílio em Sergipe da Universidade Federal de Sergipe, Arac
}

Correspondência:

Prof. Dr. Alex Vianey Callado França

Departamento de Medicina, Hospital Universitário,

Rua Cláudio Batista, s/n, CEP 49060-010 - Aracaju-SE, Brasil.

Fone: (79) 2105-1802.

E-mail: avcfranca@ufs.br

Recebido em: 24.08 .2010

Aceito em: 30.09 .2010

\section{INTRODUÇÃO}

O primeiro transplante de fígado bem sucedido da América Latina foi realizado no Brasil, no ano de 1985, no Hospital das Clínicas da Universidade de São Paulo.1 Desde então, vários outros centros passaram a realizar esse procedimento, sendo atualmente procedimento de rotina em muitos serviços hospitalares. Até o primeiro trimestre do ano de 2009 existiam 36 equipes que realizavam transplante de fígado em atividade no Brasil.2 A grande maioria dessas equipes atua na região Sudeste, a qual é responsável por cerca de $72 \%$ de todos os transplantes de fígado no Brasil. 2 No estado de Sergipe não existem equipes que realizam transplante de fígado. 3 Por essa razão, pacientes portadores de doença hepática terminal que necessitam do procedimento são encaminhados a outros estados, e suas despesas são custeadas pelo Programa de Tratamento Fora de Domicílio (TFD). 4

O TFD é um benefício estabelecido pela Portaria SAS/Ministério de Saúde no 055 de 24/02/1999 (D.O.U. de 26/02/1999, em vigor desde 01/03/1999) que consiste no fornecimento de passagens para atendimento médico especializado de diagnose, terapia ou tratamento cirúrgico de alta complexidade, a ser prestado à 
pacientes atendidos exclusivamente pelo Sistema Único de Saúde (SUS) em outros estados e entre municípios situados a mais de 50 $\mathrm{km}$ de distância. Também está previsto pagamento de ajuda de custo para alimentação e pernoite do paciente e acompanhante, somente após comprovação de tal necessidade mediante análise sócioeconômica efetuada por assistente social do município de origem do paciente. 4

O repasse do SUS para instituições que realizam transplante de fígado a partir de novembro de 2008 é de $\mathrm{R} \$ 57.089,41$ por procedimento com doador cadáver.5 Dessa forma, pacientes usuários do Programa de Tratamento Fora de Domicílio em Sergipe (TFD/SE) que foram submetidos a transplante de fígado agregaram a esse valor o custo de seus deslocamentos, gerando despesa ainda maior para a Saúde Pública do Brasil. Não há estudos locais ou nacionais para dimensionar os custos do TFD com pacientes que necessitam de transplante de fígado. Por esse motivo, faz-se necessário o conhecimento dos principais aspectos desses pacientes e seu impacto econômico para o TFD, uma vez que pode ser possível determinar novas estratégias para diminuir os gastos da saúde pública no estado de Sergipe.

\section{MÉTODOS}

Os dados foram obtidos de forma retrospectiva, a partir dos prontuários do Centro de Atenção à Saúde de Sergipe (CASE), localizado em Aracaju-SE. Foram selecionados os 55 prontuários dos pacientes atendidos pelo Programa de Tratamento Fora de Domicílio (TFD) registrados como transplante de fígado e que viajaram para avaliação pré- e pós-transplante ou para realização do mesmo em centros de referência fora do estado de Sergipe no ano de 2008.

As informações adquiridas através dos prontuários foram: gênero, data de nascimento, idade, procedência, naturalidade, ano em que o paciente ingressou no TFD, paciente já transplantado ou não ao ingressar no TFD, presença de cirrose, etiologia da cirrose, outras etiologias para a hepatopatia, indicação do transplante, ano de realização do transplante, local do serviço de avaliação/ transplante e caráter público ou privado do serviço de origem. As informações próprias dos pacientes atendidos pelo TFD em 2008 foram: número de viagens realizadas, valor das passagens, valor da ajuda de custo, dias de permanência e dias de internamento no serviço transplantador, medicações utilizadas, despesa com medicações, evolução com óbito e despesa com auxílio funeral. As informações obtidas dos acompanhantes e doadores desses pacientes foram: número de viagens, valor das passagens, valor da ajuda de custo e tempo de permanência no serviço transplantador. Todos os valores foram calculados baseados nos comprovantes de passagens e nos relatórios de ajuda de custo, considerando o valor da diário $\mathrm{R} \$ 24,751^{*}$. As medicações tiveram seus custos calculados de acordo com a tabela de valores dos medicamentos fornecida pelo Centro de Atenção à Saúde de Sergipe (CASE).

Esses dados foram analisados descritivamente e suas variáveis tiveram as frequências calculadas através do programa SPSS versão 11.5 para Windows.

O presente estudo foi desenvolvido pelo Serviço de Hepatologia do Departamento de Medicina da Universidade Federal de Sergipe, tendo sido aprovado pelo Comitê de Ética em Pesquisa sob número 0043.0.107.000-09 e segue os preceitos da declaração de Helsinque e da Resolução 196/96 do Conselho Nacional de Saúde.

\section{RESULTADOS}

Foram avaliados os 55 prontuários dos pacientes cadastrados no Programa de Tratamento Fora de Domicílio em Sergipe (TFD/ SE) para transplante de fígado, que viajaram em 2008. Dentre esses, $20(36,4 \%)$ eram do sexo feminino e $35(63,6 \%)$ do sexo masculino. A idade média foi de 30,4 anos, variando de 10 meses a 68 anos de idade. Quando analisada a faixa etária, notou-se que 15 pacientes $(27,3 \%)$ tinham idade menor ou igual a 11 anos, 34 $(61,8 \%)$ apresentaram idade entre 12 e 59 anos e havia 6 pacientes $(10,9 \%)$ com idade superior ou igual a 60 anos. A maior parte da população estudada $(50,9 \%)$ foi procedente da cidade de Aracaju/ $\mathrm{SE}$, enquanto 27 pacientes $(49,1 \%)$ relataram proceder do interior de Sergipe, não tendo sido registrado pacientes residentes em outro estado. Quanto à naturalidade, prevaleceram pacientes naturais do interior de Sergipe $(41,8 \%)$, seguidos pelos naturais de Aracaju/SE (34,6\%), sendo 13 (23,6\%) pacientes nascidos em outros estados. Em relação à presença de cirrose, constatou-se que $44(80 \%)$ desses pacientes apresentavam cirrose, cinco $(9,1 \%)$ não possuíam fígado cirrótico e $6(10,9 \%)$ não tiveram esse dado registrado no prontuário. A evolução com óbito no ano de 2008 ocorreu em sete $(12,7 \%)$ dos pacientes avaliados, sendo todos antes da realização do transplante. Na tabela 1 estão relacionados alguns dos principais aspectos dos pacientes cadastrados no TFD/ $\mathrm{SE}$, não sendo observada diferença significativa em relação ao gênero, idade, procedência, naturalidade, presença de cirrose ou ocorrência de óbito em 2008 quando comparado os grupos de pacientes transplantados ou não $(\mathrm{p}>0,05)$.

Tabela 1 - Associação entre os principais aspectos dos pacientes cadastrados no Programa de Tratamento Fora de Domicílio em Sergipe em 2008 e a realização do transplante de fígado.

\begin{tabular}{|c|c|c|c|c|}
\hline \multirow[b]{2}{*}{ Variáveis } & \multicolumn{3}{|c|}{ Realização do Transplante } & \multirow[b]{2}{*}{$p^{a}$} \\
\hline & $\begin{array}{l}\text { Sim } \\
\text { N } \%\end{array}$ & $\begin{array}{l}\text { Não } \\
\text { N } \%\end{array}$ & $\begin{array}{l}\text { Total } \\
\text { N } \%\end{array}$ & \\
\hline \multicolumn{5}{|l|}{ Gênero } \\
\hline Masculino & 1234,3 & 2365,7 & 35100,0 & \multirow{3}{*}{0,473} \\
\hline Feminino & $5 \quad 25,0$ & 1575,0 & 20100,0 & \\
\hline Total & 1730,9 & 3869,1 & 55100,0 & \\
\hline \multicolumn{5}{|l|}{ Faixa Etária } \\
\hline$\leq 11$ anos & 853,3 & $7 \quad 46,7$ & 15100,0 & \multirow{4}{*}{0,083} \\
\hline 12-59 anos & $8 \quad 23,5$ & 2676,5 & 34100,0 & \\
\hline$\geq 60$ anos & $1 \quad 16,7$ & 583,3 & $6 \quad 100,0$ & \\
\hline Total & 1730,9 & 3869,1 & 55100,0 & \\
\hline \multicolumn{5}{|l|}{ Procedência } \\
\hline Aracaju/SE & 1035,7 & 1864,3 & 28100,0 & \multirow{4}{*}{0,432} \\
\hline Interior/SE & $7 \quad 25,9$ & 2074,1 & $27 \quad 100,0$ & \\
\hline Outro estado & - & - & - & \\
\hline Total & 1730,9 & 3869,1 & 55100,0 & \\
\hline \multicolumn{5}{|l|}{ Naturalidade } \\
\hline Aracaju/SE & $6 \quad 31,6$ & 1368,4 & 19100,0 & \multirow{4}{*}{0,740} \\
\hline Interior/SE & $6 \quad 26,1$ & $17 \quad 73,9$ & 23100,0 & \\
\hline Outro Estado & 538,5 & 861,5 & 13100,0 & \\
\hline Total & 1730,9 & 3869,1 & 55100,0 & \\
\hline
\end{tabular}




\begin{tabular}{|c|c|c|c|c|}
\hline \multirow[b]{2}{*}{ Variáveis } & \multicolumn{3}{|c|}{ Realização do Transplante } & \multirow[b]{2}{*}{$\mathrm{p}^{\mathrm{a}}$} \\
\hline & $\begin{array}{l}\text { Sim } \\
\text { N } \%\end{array}$ & $\begin{array}{l}\text { Não } \\
\text { N \% }\end{array}$ & $\begin{array}{l}\text { Total } \\
\text { N \% }\end{array}$ & \\
\hline \multicolumn{5}{|l|}{ Cirrose } \\
\hline Sim & $13 \quad 29,5$ & 3170,5 & $44 \quad 100,0$ & \multirow{3}{*}{0,883} \\
\hline Não & 240,0 & 360,0 & 5100,0 & \\
\hline Total & 1530,6 & $34 \quad 69,4$ & 49100,0 & \\
\hline \multicolumn{5}{|c|}{ Óbito em 2008} \\
\hline Sim & $0 \quad 0,0$ & $7 \quad 100,0$ & $7 \quad 100,0$ & \multirow{3}{*}{0,058} \\
\hline Não & 1735,4 & 3164,6 & 48100,0 & \\
\hline Total & 1730,9 & 3869,1 & 55100,0 & \\
\hline
\end{tabular}

a Análise por provas de Qui-quadrado de Pearson.

As demais características do grupo estudado foram sintetizadas na tabela 2, onde se observou que as principais etiologias da cirrose foram Hepatite e Alcoolismo com sete $(15,9 \%)$ casos cada uma. Em seguida, foram relacionadas Atresia de Vias Biliares, a Hepatite B e Síndrome de Budd-Chiari, com $04(9,1 \%)$ casos para cada etiologia. Os outros $18(40,9 \%)$ pacientes portadores de cirrose tiveram etiologias diversas, destacando-se Hepatite Auto-imune, Doença de Wilson, Colangite Esclerosante Primária, Doença Hepática Gordurosa Não-alcóolica, Hemocromatose, Doença de Byler e outras. Dentre os 11 (20\%) pacientes que não possuíam cirrose ou cujos prontuários não especificaram tal fato, a causa mais prevalente da hepatopatia foi Atresia de Vias Biliares em $04(36,4 \%)$ casos, seguida de Síndrome de Alagille em outros $2(18,2 \%)$ casos. Os $5(57,1 \%)$ pacientes restantes tiveram etiologias diversas, como Amiloidose, Doença de Niemann-Pick, Hemangioendotelioma Infantil e Síndrome de Caroli. Os pacientes do presente estudo iniciaram o cadastro no TFD/SE principalmente nos anos de 2007 $(23,6 \%)$ e 2008 (32,7\%), anos que concentram mais da metade dos pacientes analisados; os demais $(43,7 \%)$, foram introduzidos no $\mathrm{TFD} / \mathrm{SE}$ entre os anos de 2000 e 2006 . Apenas 1 paciente $(1,8 \%)$ entre todos os avaliados, iniciou o cadastro no TFD/SE após a realização do transplante de fígado. Os pacientes analisados, em sua grande maioria $(83,6 \%)$ foram oriundos do serviço público, enquanto $9(16,4 \%)$ pacientes vieram de serviços privados. Dentre os 55 indivíduos observados, 17 (30,9\%) foram transplantados de fígado até o final do ano de 2008.

Tabela 2 - Características dos pacientes cadastrados no Programa de Tratamento Fora de Domicílio em Sergipe que viajaram em 2008.

\begin{tabular}{|c|c|c|}
\hline \multirow{2}{*}{ Variáveis } & \multicolumn{2}{|c|}{ Frequencia } \\
\cline { 2 - 3 } \multicolumn{2}{|l|}{ Etiologia da Cirrose } & $(\%)$ \\
\hline Hepatite C & 7 & $11(33,3 \%)$ \\
\hline Alcoolismo & 7 & $3(9,4 \%)$ \\
\hline Atresia de Vias Biliares & 4 & $4(12,5 \%)$ \\
\hline Hepatite B & 4 & $5(15,6 \%)$ \\
\hline Sd. de Budd-Chiari & 4 & $9(28,1 \%)$ \\
\hline Outras & 18 & $11(34,4 \%)$ \\
\hline Hepatopatia nos pacientes não-cirróticosa & \multicolumn{2}{|}{} \\
\hline Atresia de Vias Biliares & 4 & $3(23,1 \%)$ \\
\hline Sd. de Alagille & 2 & $1(7,7 \%)$ \\
\hline
\end{tabular}

\begin{tabular}{|c|c|c|}
\hline \multirow{2}{*}{ Variáveis } & \multicolumn{2}{|c|}{ Frequencia } \\
\hline & N & $(\%)$ \\
\hline Outras & 5 & $1(7,7 \%)$ \\
\hline \multicolumn{3}{|l|}{ Ano de início no TFD } \\
\hline 2008 & 18 & $(25,5 \%)$ \\
\hline 2007 & 13 & $(25,5 \%)$ \\
\hline 2006 & 5 & $(25,5 \%)$ \\
\hline 2005 & 7 & $(25,5 \%)$ \\
\hline $2004-2000$ & 12 & $(25,5 \%)$ \\
\hline \multicolumn{3}{|l|}{ Admitido no TFD } \\
\hline Pré-transplante & 54 & $(25,5 \%)$ \\
\hline Pós-transplante & 1 & $(25,5 \%)$ \\
\hline \multicolumn{3}{|l|}{ Serviço de Origem } \\
\hline Público & 46 & $(25,5 \%)$ \\
\hline Privado & 9 & $(25,5 \%)$ \\
\hline \multicolumn{3}{|l|}{ Paciente transplantado até 2008} \\
\hline Sim & 17 & 30,9 \\
\hline Não & 38 & 69,1 \\
\hline
\end{tabular}

a Pacientes que não possuíam cirrose ou cujos prontuários não relatavam cirrose.

$\mathrm{O}$ ano em que se registrou o maior número de transplantes na população estudada foi 2008 , quando ocorreram cinco $(27,8 \%)$ transplantes de fígado, todos com doador falecido, sendo um $(5,5 \%)$ deles retransplante. Em 2006 e 2007 foram realizados dois $(11,1 \%)$ transplantes de fígado em cada ano; em 2005, nenhum paciente foi transplantado, enquanto que em 2004 houve quatro $(22,2 \%)$ casos, e no período de 1999 a 2003 ocorreram os outros cinco $(27,8 \%)$ transplantes de fígado. No gráfico 1 é possível observar a distribuição dos transplantes realizados nesse grupo ao longo dos anos, no período de 1999 a 2008, destacando-se a realização de um retransplante em 2008.

Gráfico1: Número de transplantes e retransplantes de fígado realizados nos pacientes cadastrados no Programa de Tratamento Fora de Domicílio em Sergipe.

$N^{\circ}$ de Transplantes

Retransplante

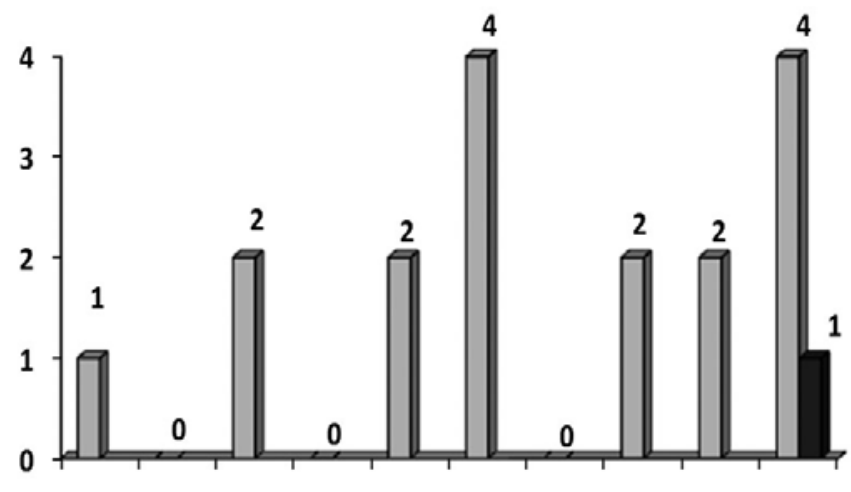

1999200020012002200320042005200620072008 
Os serviços de transplante de fígado que mais avaliaram os pacientes do TFD/SE ficavam predominantemente no estado de São Paulo, tendo sido 40 (72,7\%) pacientes avaliados nesse local. $\mathrm{Na}$ Bahia, foram avaliados $9(16,4 \%)$ pacientes e em Pernambuco, $6(10,9 \%)$ pacientes. Os estados de Minas Gerais, Paraná e Ceará foram responsáveis pela avaliação de $4(7,3 \%)$ pacientes cadastrados no TFD/SE que viajaram em 2008. Houve casos em que os pacientes foram avaliados por mais de um serviço. Quanto ao local de realização do transplante, 76,5\% dos transplantes ocorreram no estado de São Paulo, seguido de Pernambuco (11,8\%), Bahia $(5,9 \%)$ e Minas Gerais (5,9\%), não havendo transplantes realizados no Paraná e no Ceará entre os pacientes analisados. $\mathrm{O}$ gráfico 2 expressa a distribuição nos estados do Brasil onde foram realizadas avaliações de todos os pacientes estudados e onde ocorreram os transplantes de fígado.

Gráfico2: Distribuição no Brasil e entre os estados brasileiros das avaliações e dos transplantes submetidos aos pacientes cadastrados no Programa de Tratamento Fora de Domicílio em Sergipe no ano de 2008.

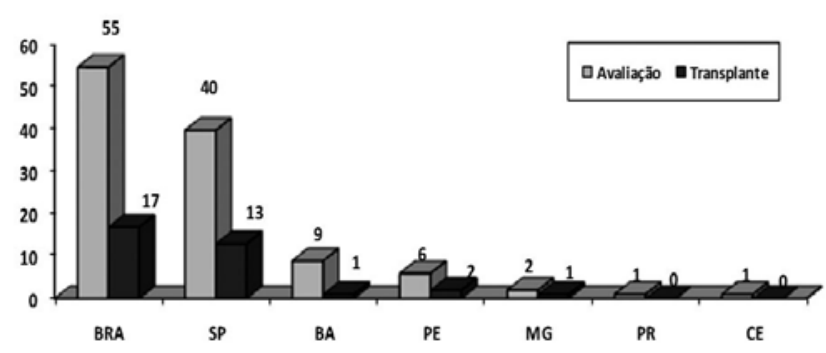

Em relação aos custos financiados pelo TDF/SE em 2008, a tabela 3 mostra os valores médios gastos com pacientes $(n=55)$, acompanhantes $(n=54)$ e doadores $(n=7)$, além do número de viagens e dos tempos de permanência e internação (este último apenas para pacientes). Os pacientes viajaram mais vezes $(4,76)$ do que os acompanhantes $(4,65)$ e doadores $(2,57)$. Apenas um $(1,8 \%)$ paciente viajou sem acompanhante em 2008. O tempo médio de permanência no serviço foi maior entre os doadores (59,86 dias), seguido dos acompanhantes (45,2 dias) e dos pacientes (44,8 dias). Os pacientes ficaram internados 14,8 $(0-174)$ dias em média. Dos 55 pacientes avaliados, 40 (72,7\%) não foram internados nos serviços de transplante de fígado de outros estados em 2008. Quanto às passagens custeadas pelo TFD/SE em 2008, os gastos foram em média de $\mathrm{R} \$ 2.892,51$ por acompanhante, $\mathrm{R} \$ 2.502,32$ por paciente e R\$ 2.243,46 por doador. A ajuda de custo teve valor médio maior entre os doadores ( $\mathrm{R} \$ 1.481,46)$, seguido dos acompanhantes (R\$ $1.136,67)$ e pacientes ( $\mathrm{R} \$ 1.105,65)$. Ao somar esses custos, avaliase que o valor gasto com pacientes foi de $\mathrm{R} \$ 198.438,35$; com os acompanhantes gastaram-se $\mathrm{R} \$ 217.575,72$ e com doadores, $\mathrm{R} \$$ 26.074,44. Desta forma, a despesa total do TFD/SE com viagens e ajuda de custo em 2008 somando pacientes, acompanhantes e doadores foi de $\mathrm{R} \$ 442.088,43$. A média de gasto dos 55 pacientes cadastrados para transplante de fígado no TFD/SE que viajaram em 2008 foi de $\mathrm{R} \$ 8.037,97$ por paciente.
Tabela 3 - Aspectos e custos dos serviços financiados pelo Programa de Tratamento Fora de Domicílio em Sergipe no ano de 2008 para pacientes, acompanhantes e doadores.

\begin{tabular}{|c|c|c|c|c|c|c|c|}
\hline \multirow{2}{*}{ Variáveis } & \multicolumn{2}{|c|}{ Paciente $(n=55)$} & \multicolumn{2}{|c|}{$\begin{array}{c}\text { Acompanhante } \\
\qquad(n=54)\end{array}$} & \multicolumn{2}{|c|}{ Doador $(\mathrm{n}=7)$} & \multirow{2}{*}{ Total $^{b}$} \\
\hline & Média & $(\%)$ & Média & $(\%)$ & Média & $(\%)$ & \\
\hline $\begin{array}{l}\text { Número de } \\
\text { viagens }^{\mathrm{a}}\end{array}$ & $\begin{array}{c}4,76 \\
(1-14) \\
\end{array}$ & 2,98 & $\begin{array}{c}4,65 \\
(1-14) \\
\end{array}$ & 2,86 & $\begin{array}{l}2,57 \\
(2-6) \\
\end{array}$ & 1,51 & - \\
\hline $\begin{array}{c}\text { Dias de } \\
\text { permanência }{ }^{a}\end{array}$ & $\begin{array}{c}44,8 \\
(3-253) \\
\end{array}$ & 52,62 & $\begin{array}{c}45,20 \\
(3-253)\end{array}$ & 53,09 & $\begin{array}{c}59,86 \\
(16-95) \\
\end{array}$ & 28,28 & - \\
\hline $\begin{array}{c}\text { Dias de } \\
\text { internação }\end{array}$ & $\begin{array}{c}14,76 \\
(0-174) \\
\end{array}$ & 35,23 & - & - & - & - & - \\
\hline $\begin{array}{l}\text { Despesa com } \\
\text { viagens }^{\text {b }}\end{array}$ & $\begin{array}{c}2.502,32 \\
(0,00- \\
8.535,57) \\
\end{array}$ & $2.086,92$ & \begin{tabular}{|c|}
$2.892,51$ \\
$(389,84-$ \\
$8.535,57)$ \\
\end{tabular} & $2.025,83$ & \begin{tabular}{|l|}
$2.243,46$ \\
$(964,04-$ \\
$5.626,08)$ \\
\end{tabular} & $1.581,28$ & $309.527,43$ \\
\hline Ajuda de custo & \begin{tabular}{|c|}
$1.105,65$ \\
$(99,00-$ \\
$6.261,75)$
\end{tabular} & $1.280,20$ & \begin{tabular}{|c|}
$1.136,67$ \\
$(99,00-$ \\
$6.261,75)$
\end{tabular} & $1.314,31$ & \begin{tabular}{|l}
$1.481,46$ \\
$(396,00-$ \\
$2.351,25)$
\end{tabular} & 699,25 & $132.561,00$ \\
\hline Custo Total ${ }^{b}$ & $198.438,25$ & - & $217.575,72$ & - & $26.074,44$ & - & $442.088,43$ \\
\hline
\end{tabular}

${ }^{a}$ Refere-se ao serviço de avaliação/transplante em outro estado ; ' $V a l o r e s$ expressos em Real $(R \$)$.

Associadas a esse valor, há ainda despesas extras com medicações, auxílio funeral (em caso de óbito do paciente em outro estado) e, excepcionalmente, UTI aérea. No gráfico 3 pode-se observar a distribuição dos valores gastos com essas despesas adicionais. Foi possível perceber que $15(27,3 \%)$ pacientes analisados necessitaram de medicações, gerando uma despesa de R \$ 65.461,38 (média de R\$ $4.364,09$ por paciente usuário de medicação). Os medicamentos mais utilizados foram os imunossupressores (Tacrolimo, Micofenolato de Mofetila, Micofenolato Sódico, Azatioprina, Ciclosporina, Mesalazina, Penicilamina e Prednisona), sendo também utilizados Eritropoetina Humana Recombinante e o Ciprofloxacino. O auxílio funeral foi necessário em dois casos, sendo que o valor gasto foi de R\$ 7.220,00. Houve ainda um caso em que a UTI aérea foi acionada, e o custo desse serviço foi de aproximadamente R\$ 67.000,00. Então, ao adicionar as despesas extras ao gasto com viagens e ajuda de custo dos 55 pacientes cadastrados para transplante de fígado no TFD/SE que viajaram em 2008, tem-se o valor médio de $\mathrm{R} \$ 10.590,49$ por paciente, totalizando o valor de $\mathrm{R} \$ 582.477,17 \mathrm{de}$ gastos para a saúde pública do Brasil.

Gráfico3: Valores gastos com despesas adicionais em pacientes cadastrados no Programa de Tratamento Fora de Domicílio em Sergipe no ano de 2008.

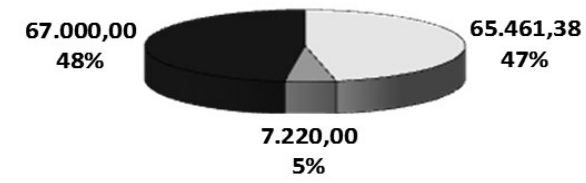

पMedicaçōes

$\square$ Auxílio Funeral

- UTI Aérea

Por fim, ao realizar análise comparativa entre os aspectos e custos dos pacientes que foram transplantados e aqueles que não foram submetidos ao transplante de fígado, notou-se que os pacientes que foram transplantados viajaram mais vezes (média de 4,94 viagens/ 
paciente), enquanto o segundo grupo viajou em média 4,68 vezes no ano de 2008. Os pacientes transplantados também permaneceram mais tempo no serviço de outro estado (média de 71,64 dias) em comparação com pacientes não-transplantados (32,79 dias). Quanto aos dias de internamento no serviço de transplante de fígado de outro estado, sete $(41,2 \%)$ dos pacientes transplantados foram internados, o que resultou em um tempo médio de internação de 20,23 dias para os pacientes desse grupo. Dentre os 38 pacientes que ainda não haviam sido submetidos ao transplante, $8(21,6 \%)$ foram internados, gerando uma média de 12,31 dias de internamento por paciente desse grupo. O custo médio com os pacientes transplantados ( $\mathrm{R} \$ 13.448,64)$, incluindo as medicações e despesas com acompanhantes e doadores, foi cerca de 1,8 vezes maior do que o custo com pacientes não-transplantados ( $\mathrm{R} \$ 7.325,73)$. As despesas que mais contribuíram para tal divergência foram: ajuda de custo e medicações, esta última com valor 40 vezes maior no grupo dos transplantados. Na tabela 4 estão expostas as comparações entre os aspectos e valores gastos com os pacientes transplantados e aqueles que não foram submetidos ao transplante de fígado.

Tabela 4 - Comparação entre os pacientes transplantados e não transplantados de fígado cadastrados no Programa de Tratamento Fora de Domicílio em Sergipe que viajaram em 2008.

\begin{tabular}{|c|c|c|c|c|c|}
\hline \multirow[t]{2}{*}{ Variáveis } & \multicolumn{2}{|c|}{$\begin{array}{l}\text { Transplantados } \\
(\mathrm{n}=17)\end{array}$} & \multicolumn{2}{|c|}{$\begin{array}{l}\text { Não-transplantados } \\
(\mathrm{n}=38)\end{array}$} & \multirow[t]{2}{*}{ Total $^{\mathrm{b}}$} \\
\hline & Média & $\mathrm{DP}( \pm)$ & Média & $\mathrm{DP}( \pm)$ & \\
\hline Número de viagens ${ }^{a}$ & $\begin{array}{c}4,94 \\
(2-10)\end{array}$ & 3,09 & $\begin{array}{c}4,68 \\
(1-14)\end{array}$ & 2,97 & - \\
\hline $\begin{array}{c}\text { Dias de } \\
\text { permanênciaa }^{\text {a }}\end{array}$ & $\begin{array}{c}71,64 \\
(6-253) \\
\end{array}$ & 72,31 & $\begin{array}{c}32,79 \\
(3-174) \\
\end{array}$ & 36,07 & - \\
\hline Dias de internaçãoa & $\begin{array}{c}20,23 \\
(0-129) \\
\end{array}$ & 40,10 & $\begin{array}{c}12,31 \\
(0-174)\end{array}$ & 33,11 & - \\
\hline $\begin{array}{c}\text { Despesa com } \\
\text { viagens }^{\mathrm{b}}\end{array}$ & $\begin{array}{c}2.717,56 \\
(0-8030,20) \\
\end{array}$ & $2.366,78$ & $\begin{array}{c}2.406,02 \\
(0-8.535,57) \\
\end{array}$ & $1.975,63$ & $137.627,50$ \\
\hline Ajuda de custo ${ }^{b}$ & $\begin{array}{l}1.757,25 \\
(148,50- \\
6.261,75) \\
\end{array}$ & $1.764,53$ & $\begin{array}{c}814,14 \\
(99,00- \\
4.306,50) \\
\end{array}$ & 873,60 & $60.810,75$ \\
\hline $\begin{array}{c}\text { Custo com } \\
\text { acompanhantes e } \\
\text { doadores }^{b}\end{array}$ & $\begin{array}{c}5.326,81 \\
(893,04- \\
10.223,48) \\
\end{array}$ & $2.733,38$ & $\begin{array}{l}4.014,47 \\
(488,84- \\
9748,32) \\
\end{array}$ & $2.222,53$ & $243.105,72$ \\
\hline $\begin{array}{l}\text { Custo com } \\
\text { medicações }^{b}\end{array}$ & $\begin{array}{c}3.647,02 \\
(0-14.151,60) \\
\end{array}$ & $4.482,41$ & $\begin{array}{c}91,10 \\
(0-2.593,80) \\
\end{array}$ & 426,85 & $65.461,38$ \\
\hline Custo Total $^{b}$ & $13.448,64$ & - & $7.325,73$ & - & $507.005,35$ \\
\hline
\end{tabular}

${ }^{a}$ Refere-se ao serviço de avaliação/transplante em outro estado ; ${ }^{b}$ Valores expressos em Real ( $R \$$ ).

\section{DISCUSSÃO}

O transplante de fígado é uma terapêutica essencial para pacientes portadores de doença hepática terminal. ${ }^{6}$ Dessa forma, fazse necessário conhecer os custos e os principais aspectos que envolvem esse procedimento. No estado de Sergipe, os pacientes que estiveram cadastrados no Programa de Tratamento Fora de Domicílio (TFD) para submeter-se à avaliação e/ou transplante em serviços de transplante de fígado de outro estado e que viajaram no ano de 2008, tiveram suas características analisadas através do presente estudo.

O gênero predominante desse grupo foi o masculino $(63,6 \%)$, enquanto apenas $36,4 \%$ dos pacientes eram do gênero feminino. Segundo o Registro Brasileiro de Transplantes (RBT), esses valores são condizentes com a população transplantada de fígado entre os anos de 1995 e 2004, onde $63 \%$ dos pacientes eram do gênero masculino e $37 \%$ do gênero feminino. ${ }^{2}$ Quanto à faixa etária, aquele mesmo estudo mostrou que $10 \%$ dos pacientes eram compostos pela população com idade superior a 60 anos, semelhante aos $10,9 \%$ de pacientes encontrados no presente estudo. A faixa etária prevalente em ambos os trabalhos foi composta por pacientes entre 12 e 59 anos. $^{2}$

$\mathrm{Na}$ literatura, há relatos de que a Hepatite $\mathrm{C}$ é a principal doença de base para transplantados de fígado (36\% dos pacientes), seguida por Atresia de Vias Biliares (11\%) e Alcoolismo (10\%). ${ }^{2}$ Neste estudo, Hepatite C (15,9\%), Alcoolismo (15,9\%) e Atresia de Vias Biliares $(9,1 \%)$ também estiveram entre as principais hepatopatias nos pacientes cirróticos. Entre os não-cirróticos prevaleceu a Atresia de Vias Biliares (36,4\%) como principal doença de base. Entre todos os transplantes de fígado realizados entre 1995 e 2004 no Brasil, $5 \%$ foram retransplantes, valor idêntico ao encontrado entre os transplantes observados neste estudo. Assim como em todo o país, onde a maior parte $(72 \%)$ dos transplantes de fígado foi realizada na região Sudeste, $82,4 \%$ de todos os transplantes deste estudo foram realizados naquela região. ${ }^{2}$

A taxa de mortalidade de pacientes em fila de espera para transplante de fígado no Brasil foi estimada em 54,4\%. ${ }^{7}$ Esse valor não foi semelhante ao encontrado neste estudo, no qual, dentre os 38 pacientes em avaliação para transplante de fígado, sete $(18,4 \%)$ faleceram. Essa discordância provavelmente deve-se ao curto tempo de avaliação dos pacientes, ou seja, apenas 12 meses.

Observa-se que a maioria dos transplantes foi realizada em 2006, 2007 e 2008, o que coincide com a implantação do serviço de hepatologia do Hospital Universitário da UFS, que avalia tanto adultos quanto crianças. Esse serviço é o único de referência para atendimento de pacientes portadores de doenças hepáticas no Estado de Sergipe, atendendo apenas usuários do SUS.

Outros aspectos despertaram atenção no presente estudo, como o fato de o gasto total com os acompanhantes ( $\mathrm{R} \$ 217.575,72)$ ser maior do que o gasto total com os pacientes ( $\mathrm{R} \$ 198.438,25)$. Tal ocorrência pode ser devido a um número considerável de pacientes crianças, o que reduz o valor das tarifas para as passagens. Além disso, deve-se destacar que os pacientes não recebem o benefício da ajuda de custo durante o período de internação. Um fator que também pode ter contribuído para tal foi a ocorrência de apenas 1 $(1,8 \%)$ paciente ter viajado sem acompanhante, o que ainda gerou um tempo médio de permanência maior para os acompanhantes (45,2 dias) do que para pacientes (44,8 dias). O número de pacientes $(15=27,3 \%)$ que utilizaram medicamentos fornecidos pelo CASE também foi abaixo do esperado, uma vez que os 17 pacientes $(30,9 \%)$ transplantados deveriam fazer uso de medicações. Uma explicação plausível para tal discordância pode ser o fornecimento de medicações no serviço de avaliação/transplante de outro estado. O custo do transplante de fígado para o Sistema Único de Saúde do Brasil a partir de novembro de 2008 foi de $\mathrm{R} \$ 57.089,41$ por procedimento. ${ }^{5}$ Dessa forma, as despesas médias geradas pelos pacientes avaliados em nossos estudos de $\mathrm{R} \$ 10.590,49$ por paciente (incluindo os gastos adicionais) aumentaram em mais de $18 \%$ o custo do transplante de fígado. A maior parte desse valor foi gasta com 
serviços indispensáveis aos pacientes que necessitam de transplante de fígado. Contudo, poderia haver uma grande redução desse custo com a implantação e capacitação da equipe de saúde no próprio estado de Sergipe para realizar avaliações prévias e posteriores ao transplante. Com a implantação da referida equipe, seria possível diminuir o número de viagens, promovendo melhora da qualidade de vida desses pacientes, bem como redução de custo para o serviço público. Essa constatação torna-se ainda mais presente em pacientes que já realizaram transplante de fígado, pois estes apresentam custo médio ainda maior ( $\mathrm{R} \$ 13.448,64$ por paciente transplantado), sendo suas viagens mais frequentes, maior o tempo médio de permanência e internação em serviços de transplante de fígado de outros estados e maiores os custos com viagens, ajuda financeira, despesas com acompanhantes e medicações.

A implantação da estrutura necessária para realização do transplante no próprio estado de Sergipe também poderia tornar-se uma estratégia para restringir os gastos com o TFD em Sergipe.

\section{CONCLUSÃO}

Ao analisar os 55 prontuários dos pacientes cadastrados no TFD/ SE para transplante de fígado que viajaram em 2008, constatou-se que, naquele ano, houve um gasto com deslocamento e medicações correspondente a mais de $18 \%$ do valor do procedimento de transplante de fígado. Esse gasto adicional poderia ser reduzido através da implantação de novas estratégias para diminuir os gastos da saúde pública no estado de Sergipe.

\section{ABSTRACT}

Introduction: In Brazil, liver transplantation is performed mainly in the Southeast region. In states where there is no liver transplantation service, such as Sergipe, patients of the National Health System are driven to other states, and such expenses are funded by the Out of Home Treatment Program (TOH). Purpose: The aim of this study was to set main aspects of patients referred to liver transplantation and the costs of services funded by TOH in Sergipe along 2008. Methods: Analysis of 55 records of patients who were registered in TOH in Sergipe during 2008. Age, gender, origination, place of birth of the patients, presence of cirrhosis and death in 2008 were assessed. Carers, donors and patients' features and their costs were compared, as well as transplanted versus non-transplanted patients. Some additional costs of those patients were also analyzed. Results: Most patients were men (63.6\%), with ages between 12 and 59 (61.8\%) from Aracaju/ SE (50.9\%), and 49.1\% came from the country area of Sergipe. About $80 \%$ of patients presented cirrhosis, and $12.7 \%$ died along 2008 . The main causes of cirrhosis were hepatitis $\mathrm{C}$ and alcoholism, both in $15.9 \%$ of cases. Liver transplantation was performed in $30.9 \%$ of patients, and $76.5 \%$ of those transplantations were performed in São Paulo. Carers caused higher TOH expenditures. R\$ $217,575.72$ from a total amount of R \$ 442,088.43 included patients and donors. The average cost of each patient funded by TOH was R $8,037.97$ or R $\$$ $10,590.49$, with additional costs included. Patients traveled an average of 4.76 times in 2008 , and the transplanted patients spent 1.8 times more than non-transplanted patients. Conclusion: Hepatic cirrhosis was the major cause for the TOH indication. It was concluded that the cost of the program was around $\mathrm{R} \$ 8,000.00$ per patient in 2008. Based on this figure, a pre- and post-liver transplantation service could be created at the state level.

Keywords: Liver Transplantation, Health Expenditures, Single Health System.

\section{REFERÊNCIAS}

1. Mies S. Transplante de Fígado [revisão]. Rev Ass Med Brasil 1998; 44(2): 127-34.

2. Associação Brasileira de Transplante de Órgãos [homepage na internet]. Registro Brasileiro de Transplantes: RBT 10 anos - Análise Qualitativa 2007 [acesso em 14 de junho de 2009]. Disponível em: http://www.abto.org.br/abtov02/portugues/ profissionais/rbt/rbt10anos/index.aspx?idCategoria $=2$

3. Marinho A, Cardoso SS, Almeida VV. Os Transplantes de Órgãos nos Estados Brasileiros [texto para discussão $n^{\circ}$ 1317]. Instituto de Pesquisa Econômica Aplicada [homepage na internet], c 2006 [acesso em 2009 Maio 12]. Disponível em: http:// www.ipea.gov.br/sites/000/2/publicacoes/tds/td_1317.pdf

4. Secretaria de Atenção à Saúde (SAS). Brasília (DF): Ministério da Saúde. [acesso em 2009 Jun. 16]. Portaria SAS nº 055. Disponível em: http://tr2001.saude.gov.br/ sas/PORTARIAS/Port99/PT-055.html

5. Secretaria de Atenção à Saúde (SAS). Brasília (DF): Ministério da Saúde. [acesso em 2009 Jun. 16]. Portaria GM nº 2.041. Disponível em: http://dtr2001.saude.gov. br/sas/PORTARIAS/Port2008/GM/GM-2041re.htm

6. National Institute of Health Consensus Development Conference Statement: liver transplantation - June 20-23, 1983. Hepatology. 1984;4(suppl):107-10.

7. Marinho A. Um estudo sobre as filas para transplantes no Sistema Único de Saúde brasileiro. Cad. Saúde Pública, Rio de Janeiro. 2006 out;22(10):2229-39. 